\title{
PERFORMANCE COMPARISION OF DIFFERENT ROUTING PROTOCOLS WITH FUZZY INFERENCE SYSTEM IN MANET
}

\author{
Subhrananda Goswami', Subhankar Joardar ${ }^{2}$, Chandan Bikash Das ${ }^{3}$
}

1 Department of IT, Global Group of Institutions, Haldia, Purba Medinipur, WB, India, e-mail: subhrananda_ usca@yahoo.co.in

2 Department of Computer Sciences \& Engineerig, Haldia Institute of Technology, Haldia, Purba Medinipur, WB, India, e-mail: subhankarranchi@yahoo.co.in

${ }^{3}$ Department of Mathematics, Tamralipta Mahabidalay, Tamluk, Purba Medinipur, WB, India, e-mail: cdas_ bikash@yahoo.co.in

Received: 2015.05.30

Accepted: 2015.08.05

Published: 2015.09.01

\begin{abstract}
An ad hoc wireless network consists of mobile networks which create an underlying architecture for communication without the help of traditional fixed-position routers. There are different protocols for handling the routing in the mobile environment. Routing protocols used in fixed infrastructure networks cannot be efficiently used for mobile ad-hoc networks (MANET), so it requires different protocols. The node moves at different speeds in an independent random form, connected by any number of wireless links, where each node is ready to pass or forward both data and control traffic unrelated to its own use ahead (routing) to other nodes in a flexible interdependence of wireless communication in between. In contrast to infrastructure wireless networks, where the communication between network nodes is take place by a special node known as an access point. It is also, in contrast to wired networks in which the routing task is performed by special and specific devices called routers and switches. In this paper, we consider fuzzy inference system, an attempt has been made to present a model using fuzzy logic approach to evaluate and compare three routing protocols i.e. AODV, DSDV and DSR using effective factor of the number of nodes based on 3 outputs of control overhead, delay and PDR (totally fuzzy system with 4 outputs) in order to select one of these two routing protocols properly under different conditions and based on need and goal. To show efficiency and truth of fuzzy system, three protocols have been evaluated equally using NS-2 simulator and attempt has been made to prove efficiency of the designed fuzzy system by comparing results of simulation of fuzzy system and NS-2 software.
\end{abstract}

Keywords: MANET, AODV, DSDV, DSR, expert system, control overhead, delay, PDR, NS-2 software.

\section{INTRODUCTION}

A Mobile Ad hoc NETwork (MANET) is a kind of wireless ad-hoc network, and is a selfconfiguring network of mobile routers (and associated hosts) connected by wireless links - the union of which forms an arbitrary topology. The routers are free to move randomly and organize themselves arbitrarily; thus, the network's wireless topology may change rapidly and unpredict- ably. Such a network may operate in a standalone fashion, or may be connected to the Internet. The surrounding physical environment significantly attenuates and distorts the radio transmissions since signal quality degrades with distance. Because of limited transmission area of these nodes, the effective throughput may be less than that of node's maximum transmission capacity. Hence, it may be needed for one mobile node to take the assistance of other nodes in forwarding its packets 
to the desired destination. A node can move anytime in an ad hoc scenario and, as a result, such a network needs to have routing protocols which can adapt dynamically changing wireless topology. However, since there is no fixed infrastructure in a network, each mobile node operates not only as a node but also as a router, forwarding packets from one node for other mobile nodes in the network, that may not be within direct wireless transmission range of each other $[1,2]$. Some of these protocols have been studied and their performances have been analysed in detail. Broch et al. [3] evaluated four protocols using mobility and traffic scenarios similar to those we used. They focused on packet loss, routing message overhead and route length. In Johansson et al. [4], compare three routing protocols, over extensive scenarios, varying node mobility and traffic load. They focus on packet loss, routing overhead, throughput and delay, and introduce mobility measures in terms of node relative speed. Finally, in Das et al. [5], compare the performance of two protocols, focussing on packet loss, packet end to end delay and routing load. They obtained simulation results consistent with previous works and conclude' with some recommendations for improving protocols.

This paper discusses in detail, analyzes and evaluates the functioning ofAODV, DSR and DSDV with fuzzy logic, and NS-2 and how well it adapts to the dynamically changing link conditions. In this paper, the designed fuzzy system, results of simulation are mentioned with NS-2 software and at the end, result of the research is mentioned.

\section{ROUTING PROTOCOLS}

\section{Ad-hoc On-demand Distance Vector protocol (AODV)}

Ad-hoc On-demand Distance Vector protocol (AODV) protocol is taken from the RFC [5]. AODV is a very simple, efficient, and effective routing protocol for mobile ad-hoc networks which do not have fixed topology. This algorithm was motivated by the limited bandwidth that is available in the media that are used for wireless communications. The route discovery is used by broadcasting the RREQ message to the neighbors with the requested destination sequence number, which prevents the old information to be replied to the request and also prevents looping problem, which is essential to the traditional distance vector protocols [6]. The route request does not add any new information about the passed hosts but only increases its hop metric. Each passed host makes update in their own routing table about the requested host. This information helps the destination reply to be easily routed back to the requested host. The route reply use RREP message that can be only generated by the destination host or the hosts which have the information that the destination host is alive and the connection is fresh.

\section{Dynamic Source Routing protocol (DSR)}

The Dynamic Source Routing protocol (DSR) $[7,8]$ is a simple and efficient routing protocol designed specifically for use in multi-hop wireless ad hoc networks of mobile nodes. Using DSR, the network is completely self-organizing and self-configuring, requiring no existing network infrastructure or administration. Network nodes cooperate to forward packets for each other to allow communication over multiple "hops" between nodes not directly within wireless transmission range of one another. As nodes in the network move about or join or leave the network, and as wireless transmission conditions such as sources of interference change, all routing is automatically determined and maintained by the DSR routing protocol. Since the number or sequence of intermediate hops needed to reach any destination may change at any time, the resulting network topology may be quite rich and rapidly changing.

\section{Destination Sequenced Distance Vector protocol (DSDV)}

Destination Sequenced Distance Vector routing (DSDV) is adapted from the conventional Routing Information Protocol (RIP) to ad hoc networks routing. It adds a new attribute, sequence number, to each route table entry of the conventional RIP. The Perkins and Bhagwat developed this routing protocol in 1994. DSDV is a proactive hop-by-hop distance vector routing protocol, requiring each node to broadcast routing updates periodically. It is based on modified bellman ford routing algorithm with some enhancement to calculate path [9]. In DSDV, each node maintain routing information which stores address of the next hop, cost matrix towards each destination, sequence number which is created by the destination node. The cost matrix is used for hop count, by which we can determine how many 
number of hops it takes for the packet to reach the destination. The "full dump" and "incremental update" is two ways in DSDV for sending information of routing table updates. As the name "full dump" implies, the complete routing table is send in update message while incremental update contains only the entries with metric that have been changed since last update was sent. DSDV protocol guarantees loop free paths and count to infinity problem is reduced in DSDV [10]. On the contrary in DSDV there is wastage of bandwidth due to unnecessary advertising of routing information even if there is no change in the network topology [11] neither does DSDV support multi path routing. It is difficult to determine a time delay for the advertisement of routes [12].

\section{FUZZY SYSTEM}

Fuzzy systems are able to make decision and control a system with expert systems so that the most applicable case for using them is to model relations in complex medium or anywhere which there is no clear model in the system such that it makes conclusion and decision for the system by relying on some inputs and their results. It is very complex to recognize the reasons for efficiency of a test technique.

In various applications and engineering scenarios there will be a need to "defuzzify" the fuzzy results we generate through a fuzzy systems analysis. In other words, we may eventually find a need to convert the fuzzy results to crisp results. For example, in classification and pattern recognition. We may want to transform a fuzzy partition or pattern into a crisp partition or pattern; in control we may want to give a single-valued input to a semiconductor device instead of a fuzzy input command. This "defuzzification" has the result of reducing a fuzzy set to a crisp singlevalued quantity, or to a crisp set; of converting a fuzzy matrix to a crisp matrix; or of making a fuzzy number a crisp number.

Mathematically, the defuzzification of a fuzzy set is the process of "rounding it off" from its location in the unit hypercube to the nearest (in a geometric sense) vertex. If one thinks of a fuzzy set as a collection of membership values, or a vector of values on the unit interval, defuzzification reduces this vector to a single scalar quantity presumably to the most typical (prototype) or representative value.
The following Figure shows general diagram of MANET model with fuzzy system. The most important idea in the use of fuzzy system which has been shown in Figure 1 is that verbal words are transferred to fuzzy system and the fuzzy system expresses the efficiency of the protocols under different conditions considering the signs which have been shown with verbal words.

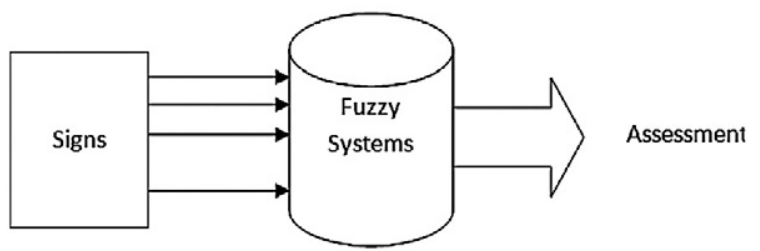

Fig. 1. Function of fuzzy system

\section{Fuzzy Inference System (FIS)}

Hayashi et al. [15] showed that a feed forward neural network could approximate any fuzzy rule based system and any feed forward neural network may be approximated by a rule based fuzzy inference system . Fusion of Artificial Neural Networks (ANN) and Fuzzy Inference Systems (FIS) have attracted the growing interest of researchers in various scientific and engineering areas due to the growing need of adaptive intelligent systems to solve the real world problems [13, 14]. A neural network learns from scratch by adjusting the interconnections between layers. Fuzzy inference system is a popular computing framework based on the concept of fuzzy set theory, fuzzy if-then rules, and fuzzy reasoning. The advantages of a combination of neural networks and fuzzy inference systems are obvious [16]. The analysis reveals that the drawbacks pertaining to these approaches seem complementary and therefore it is natural to consider building an integrated system combining the concepts.

In the fuzzy system, we have used rules as equation (1) to model the concepts:

$$
\text { if } x_{1} \text { is } A_{1, \ldots,}^{1} x_{m} \text { is } A_{m}^{1} \text { then } y=B^{1}
$$

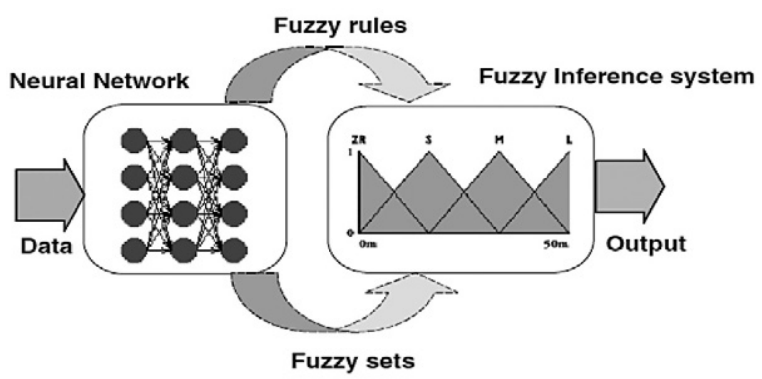

Fig. 2. Fuzzy Inference System model 
The most paramount reasons justifying use of fuzzy systems are Annabelle Mercier [2005], Kim-Hui Yap [2005]:

- The sophistication of natural world which leads to an approximate description or a fuzzy system for modeling.

- Necessity of providing a pattern to formulate mankind knowledge and applying it to the actual systems.

Thus, the following procedure is considered to define expert fuzzy system:

- Defining input-output sets which accept normalized input-output pairs.

- Generating if-else fuzzy rules based on inputoutput pairs.

- Creating fuzzy rule base.

- Implementing fuzzy system based on fuzzy rules.

In our inference engine we also used Mamdani product implication and individual-rule based inference combined with algebraic summation and multiplication for t-norms and max for s-norms. Thus, product inference engine can be written as denoted by equation (2):

$\mu_{B^{1}}(y)=\max _{l-1}^{n}\left[\sup \left(\mu_{A^{1}}(x) \cup_{i=1}^{k} \mu_{A_{i}^{l}}\left(x_{i}\right) \mu_{B^{1}}(y)\right)\right]$

Center average defuzzifier is calculated as shown in equation (3):

$$
S\left(A_{j} \Rightarrow \text { Classh }\right)=\frac{1}{\mathrm{~m}} \sum_{\mathrm{x}_{\mathrm{p}} \in \text { Classh }} \mu_{\mathrm{A}_{\mathrm{j}}}\left(\mathrm{x}_{\mathrm{p}}\right)
$$

\section{Parameters of fuzzy system}

The learning algorithm uses a gradient descent procedure that uses an error measure $E$ (difference between the actual and target outputs) to fine-tune the parameters of the membership functions (MF). The procedure is very similar to the delta rule for multilayer perceptions. The learning takes place in an offline mode. For the input vector, the resulting error $E$ is calculated and based on that the consequent parts (a real value) are updated. Then the same patterns are propagated again and only the parameters of the MFs are updated. This is done to take the changes in the consequents into account when the antecedents are modified. A severe drawback of this approach is that the representation of the linguistic values of the input variables depends on the rules they appear in. Initially identical linguistic terms are represented by identical membership functions.
A Mamdani neuro-fuzzy system uses a Supervised learning technique (back propagation learning) to learn the parameters of the membership functions. The detailed function of each layer is as follows:

- Layer-1(input layer): No computation is done in this layer. Each node in this layer, which corresponds to one input variable, only transmits input values to the next layer directly. The link weight in layer 1 is unity.

- Layer-2 (fuzzification layer): Each node in this layer corresponds to one linguistic label (excellent, good, etc.) to one of the input variables in layer 1 . In other words, the output link represent the membership value, which specifies the degree to which an input value belongs to a fuzzy set, is calculated in layer 2 . A clustering algorithm will decide the initial number and type of membership functions to be allocated to each of the input variable. The final shapes of the MFs will be fine-tuned during network learning, layer 2. A clustering algorithm will decide the initial number and type of membership functions to be allocated to each of the input variable. The final shapes of the MFs will be fine-tuned during network learning.

- Layer-5 (combination and defuzzification layer): This node does the combination of all the rules consequents using a T-conorm operator and finally computes the crisp output after defuzzification.

The simulation of the Fuzzy inference system was done using MATLAB and the values are obtained. In FIS, we use 1 factor of the number of nodes has been used in this system for evaluation of three AODV, DSDV and DSR routing protocols as input parameter and based on this input factor, effect of the factor on three AODV, DSDV and DSR routing protocols is studied but, as mentioned above, other factors, such as nodes searching speed, number of packets etc. are also effective on evaluation of three AODV, DSDV and DSR routing protocols. In this paper, FIS tools were used in Matlab software to determine efficiency of test technique and its general diagram is shown in Figure 3. This system has 1 input field which relates to factor affecting evaluation of three AODV,DSDV and DSR routing protocols and three classes i.e. min, normal and max verbal words have been assigned to each factor and 3 output fields which show efficiency of three 
AODV, DSDV and DSR routing protocols and the output has been classified into three groups and low, normal and high verbal words have been assigned to each factor. In Figures 4 and 5, one of the membership functions of input and output parameters is shown.

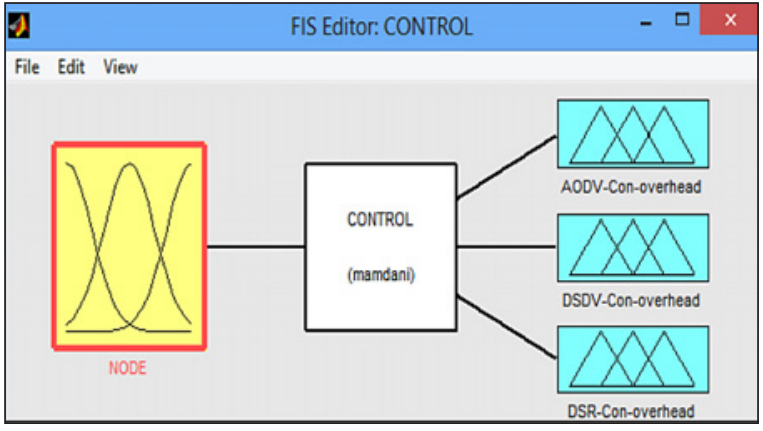

Fig. 3. General model of fuzzy expert system for evaluation of three routing protocols

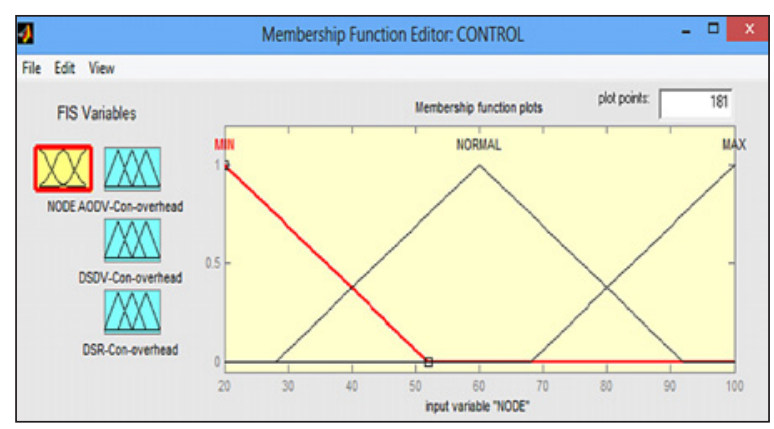

Fig. 4. Membership function relating to input of the number of node

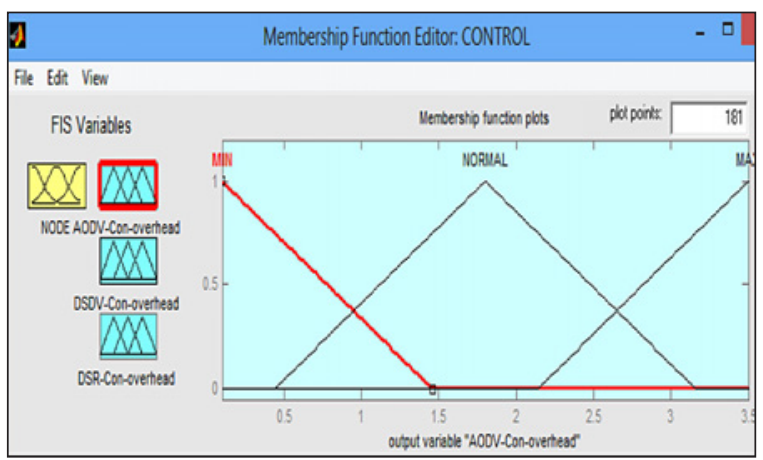

Fig. 5. Membership function relating to control overhead of AODV routing protocol

\section{Fuzzy if-then rules}

We write if-them rules as follows:

\section{Control overhead}

1) If (Node is min) then (AODV-con-overhead is min) (DSDV-con-overhead is min) (DSR-conoverhead is min).
2) If (Node is normal) then (AODV-con-overhead is min) (DSDV-con-overhead is normal) (DSR-con-overhead is min).

3) If (Node is max) then (AODV-con-overhead is normal) (DSDV-con-overhead is min) (DSRcon-overhead is max).

\section{PDR}

1) If (Node is min) then (AODV-PDR is max) (DSDV-PDR is min) (DSR-PDR is normal).

2) If (Node is normal) then (AODV-PDR is max) (DSDV-PDR is min) (DSR-PDR is normal).

3) If (Node is max) then (AODV-PDR is normal) (DSDV-PDR is min) (DSR-PDR is normal).

\section{Simulations results of fuzzy system}

We use MATLAB software which is a suitable medium for simulation of such systems has been used. Simulation of two cases of tests with 20 and 40nodes is given in Figures 6 and 7.

\section{Control overhead}

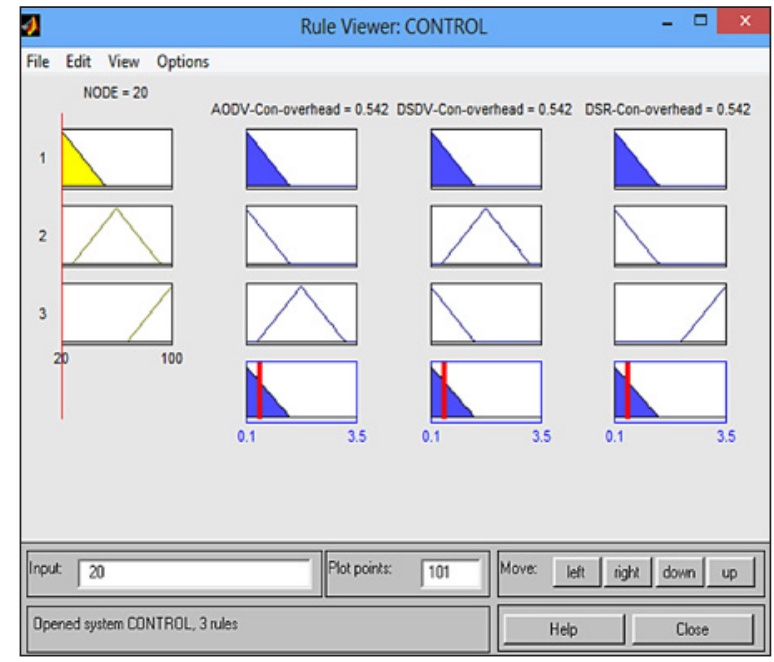

Fig. 6. Results of simulation with 20 nodes

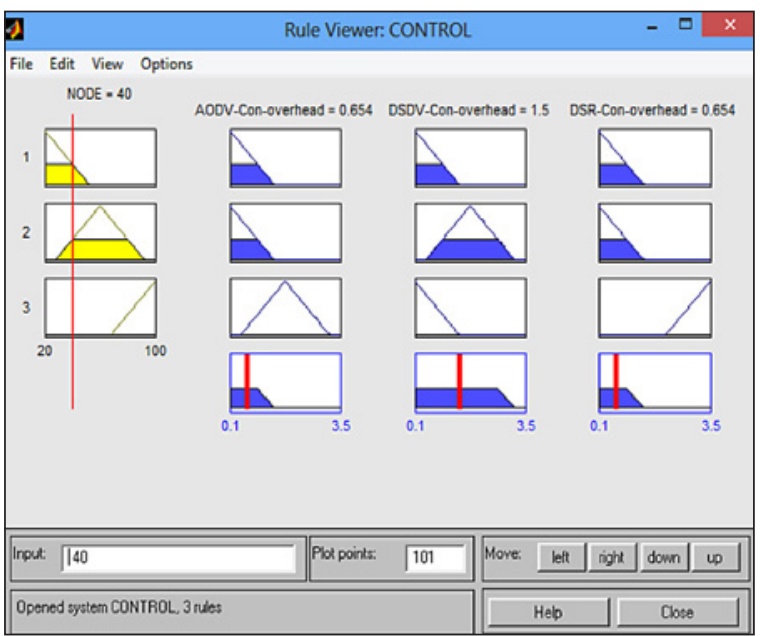

Fig. 7. Results of simulation with 40 nodes 


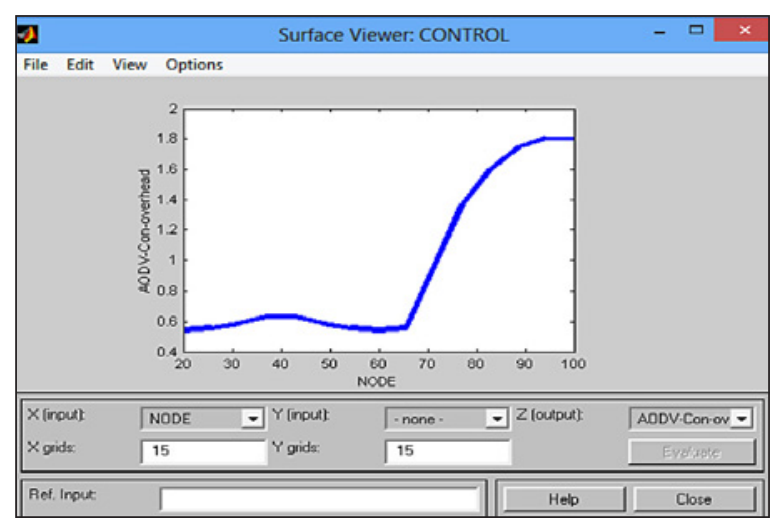

Fig. 8. Effect of number of node on output of control overhead in AODV protocol

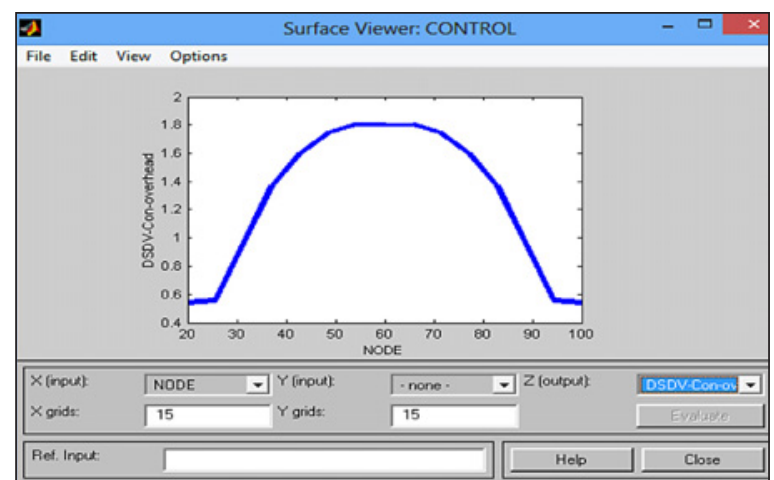

Fig. 9. Effect of number of node on output of control overhead in DSDV protocol

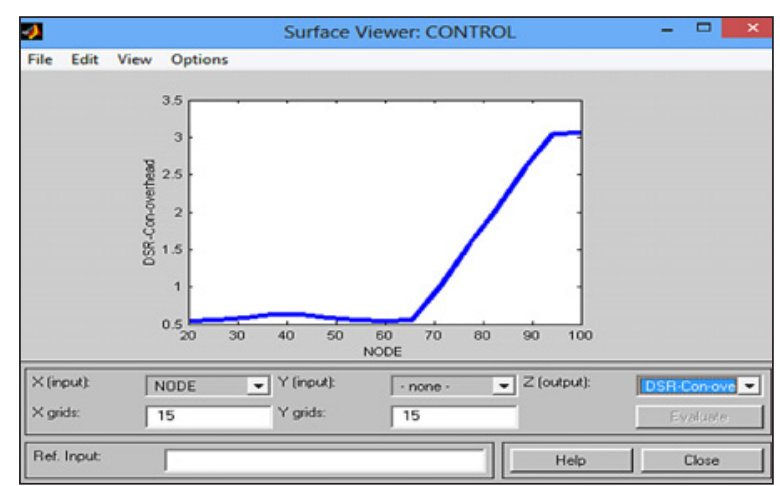

Fig. 10. Effect of number of node on output of control overheadin DSR protocol

\section{PDR}

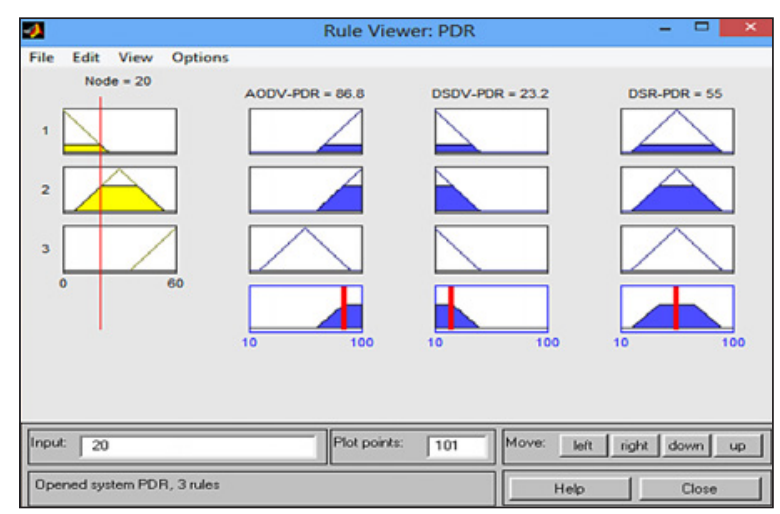

Fig. 11. Results of simulation with 20 nodes

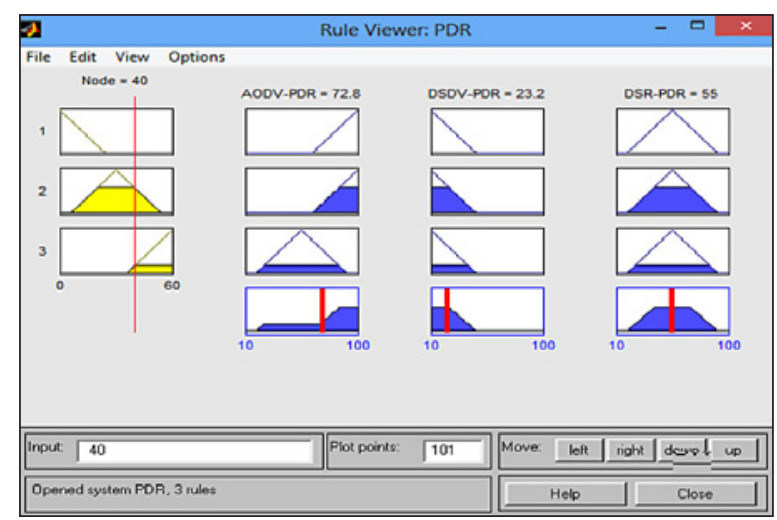

Fig. 12. Results of simulation with 40 nodes

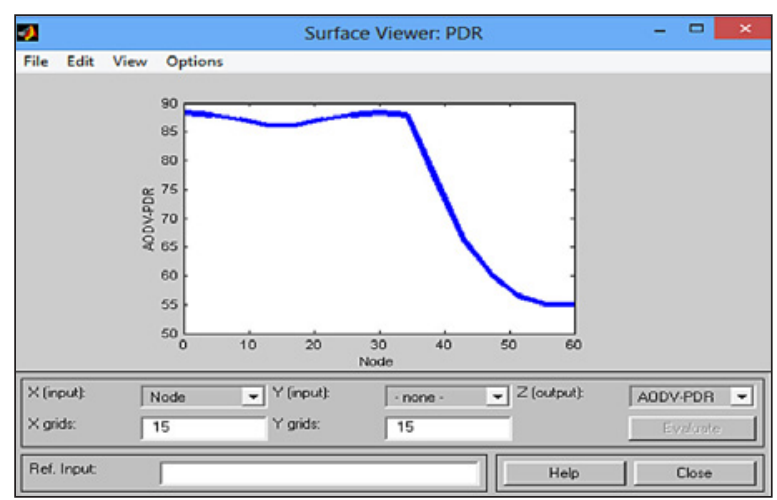

Fig. 13. Effect of number of node on output of PDR in AODV protocol

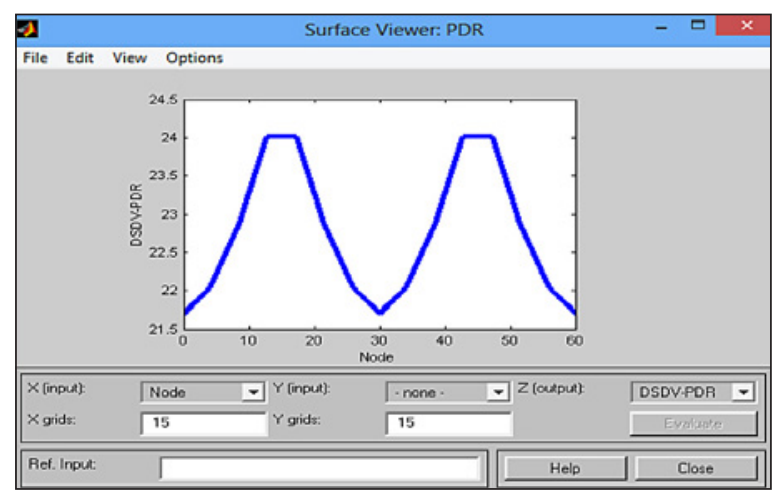

Fig. 14. Effect of number of node on output of PDR in DSDV protocol

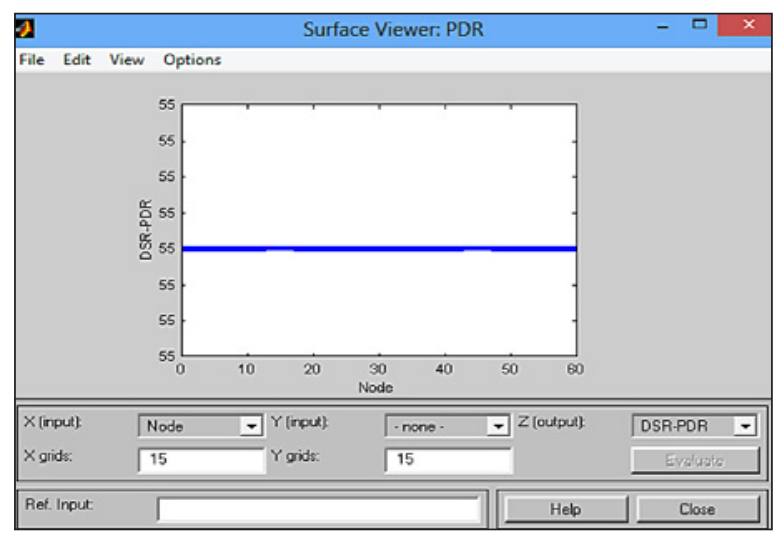

Fig. 15. Effect of number of node on output of PDR in DSR protocol 
Results obtained from execution of the designed fuzzy system for the number of different nodes are exactly mentioned in the above figure. Now, we have evaluated and simulated AODV, DSDV and DSR routing protocols for the number of similar nodes with NS-2 software in order to show performance and reliability of the proposed fuzzy system by comparing results of executing fuzzy system and NS-2 software with each other.

\section{SIMULATION RESULTS WITH NS-2 SOFTWARE}

In order to analyze and compare the performance of the three routing protocols AODV, DSR and DSDV, simulation experiments were performed. The purpose of the simulations was to compare the efficiency of the routing protocols based on two simulation parameters.

Table 1. Simulation environment

\begin{tabular}{|l|l|}
\hline Parameter & Values \\
\hline Simulator & NS2 (Version 2.34) \\
\hline Channel type & Channel/Wireless Channel \\
\hline Radio-propagation model & Propagation/Two Ray Ground \\
\hline Network interface type & Phy/Wireless Phy \\
\hline MAC type & Mac/802.11 \\
\hline Interface queue type & Queue/Drop Tail/Pri Queue \\
\hline Link layer type & LL \\
\hline Antenna model & Antenna/Omni Antenna \\
\hline Maximum packet in ifq & 50 \\
\hline Area (M×M) & 800 \\
\hline Source type & CBR \\
\hline Routing protocol & DSR, DSDV, AODV \\
\hline
\end{tabular}

\section{Control over head}

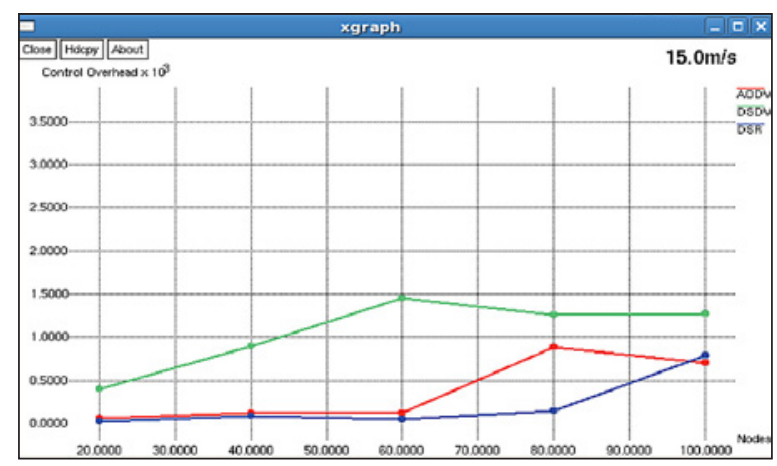

Fig. 16. Control overhead at $15 \mathrm{~m} / \mathrm{s}$

Explanation: At $15 \mathrm{~m} / \mathrm{s}$ AODV and DSR show a good result.

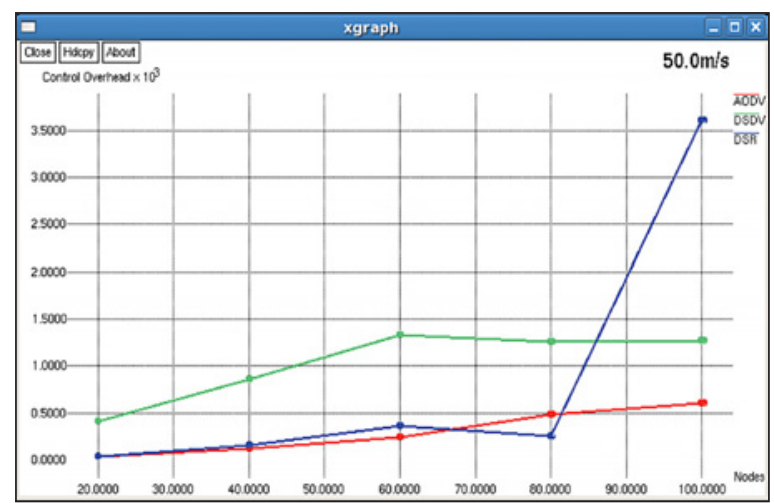

Fig. 17. Control overhead at $50 \mathrm{~m} / \mathrm{s}$

Explanation: At $50 \mathrm{~m} / \mathrm{s}$ AODV gives better result than DSR and DSDV.

\section{PDR}

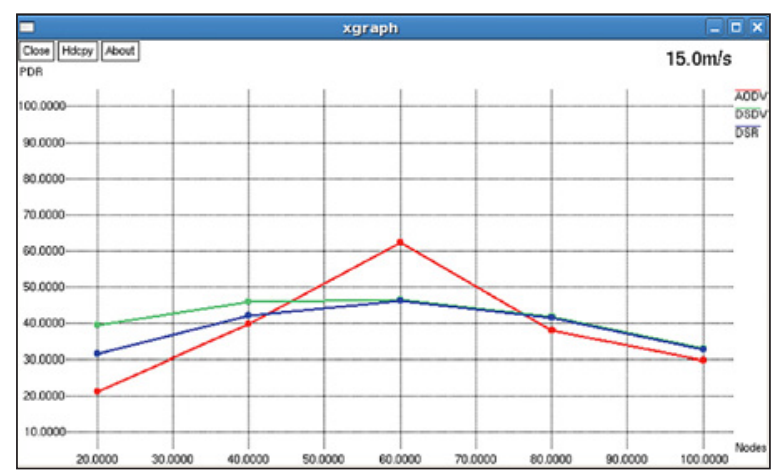

Fig. 18. Packet delivery ratio at $15 \mathrm{~m} / \mathrm{s}$

Explanation: At $15 \mathrm{~m} / \mathrm{s}$ DSR and DSDV shows approximately the same behavior but DSR shows steady

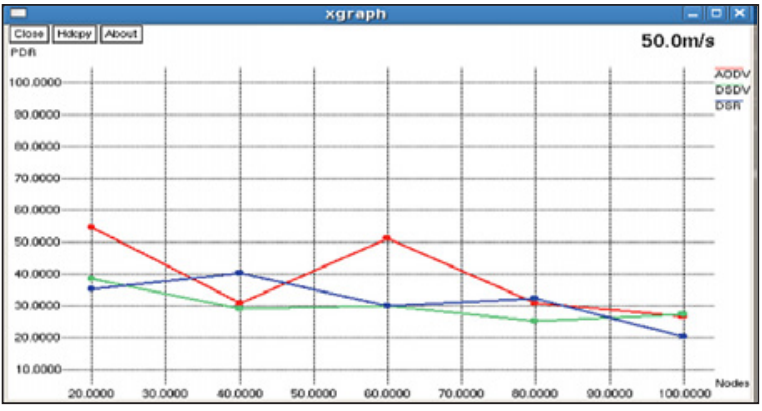

Fig. 19. Packet delivery ratio at $50 \mathrm{~m} / \mathrm{s}$

Explanation: At $50 \mathrm{~m} / \mathrm{s}$ AODV is best and in the long run it shows very good results.

\section{CONCLUSIONS}

We have presented a self-healing technique based on fuzzy concepts for mobile ad-hoc networks. We evaluate DSDV, DSR and AODV protocols in mobile ad hoc network and to prove truth of the fuzzy system, we compare the results of comparing two protocols with NS-2 software and the results show that the designed fuzzy system 
has suitable efficiency for proposing and selecting one of these three routing protocols principally and logically under different conditions and based on different applications. The basic idea is to modify the entries of the neighbor table and the time-stamp of the entry each based on the fuzzy system. The present system has only two inputs. The performance may be improved, if we consider more than two metrics and have more rules to make a perfect decision. It can be generally said that AODV protocol has better performance than the DSDV and DSR protocol in terms of the data transfer rate per second and delay rate with increasing the number of node in the network.

\section{REFERENCES}

1. Boukerche A.: Performance evaluation of routing protocols for ad hoc wireless networks. Kluwer Academic Publishers, Netherlands, Mobile Networks and Applications, 9(4), 2004, 333-342.

2. Boukerche A., Bamis A., Chatzigiannakis I., Nikoletseas S.: A mobility aware protocol synthesis for efficient routing in ad hoc mobile networks. The International Journal of Computer and Telecommunications Networking, 52(1), 2008, 130-154.

3. Broch J., Maltz A.D., Johnson D.B., Hu Y.C., Jetcheva J.: A performance comparison of multihop wireless ad hoc networks. In: Proceedings of the 4th Int. Conjkrence on Mobile Computing and Networking (ACM MOBICOM'98), 1998, 85-97.

4. Broch J., Maltz A.D., Johnson D.B.: The dynamic Source routing protocol for mobile ad hoc networks. lntemet Draft, MANET Working Group, 1999.

5. Perkins C.E., Belding-Royer E.M., Das S.R.: Ad hoc on-demand distance vector routing. In: Charles E. Perkins (Ed.) IEFT MANET Draft, Ad Hoc Networking, 2002.

6. Hong X., Xu K., Gerla M.: Scalable routing protocols for mobile ad hoc networks. Network, IEEE, 16(4), 2002, 11-21.

7. Maltz D.A. and Johnson D.B.: Dynamic source routing in ad hoc wireless networks. In: Mobile Computing, Kluwer Academic Publishers, 1996, $153-181$.

8. Johnson D.B. : Routing in ad hoc networks of mobile hosts. In: Proceedings of the IEEE Workshop on Mobile Computing Systems and Applications, IEEE Computer Society, 1994, 158-163.

9. Maan F. and Mazhar N.: MANET routing protocols vs mobility models: A performance evaluation. In: Proceedings of IEEE, 2011.

10. Lu Y., Wang W., Zhong Y., Bhargava B.: Study of distance vector routing protocols for mobile ad hoc networks. In: Proceedings of the First IEEE International Conference on Pervasive Computing and Communications (PerCom'03), 2003, 187-194.

11. Baumung P., Zitterbart M., Kutzner K.: Improving delivery ratios for application layer multicast in mobile ad-hoc network. Journal of Computer Communication, 28(14), 2005, 1669-1679.

12. Bein D., Datta K.A., Jagganagari R.C., Villain V.: A self-stabilizing link - cluster algorithm in mobile ad hoc networks. In: Proceedings of The International Symposium on Parallel Architectures, Algorithms and Networks (I-SPAN), 4(2), 2005, 436-441.

13. Andlinger P. and Reichl E.R.: Fuzzy-neunet: A non-standard neural network. In: Prieto et al. (Ed.), 1991, 173-180.

14. Arao M., Fukuda T., Shimokima K., Flexible intelligent system based on fuzzy neural networks and reinforcement learning. In: Proceedings of IEEE International Conference on Fuzzy Systems, 5(1), 1995, 69-70.

15. Hayashi Y. and Buckley J.J. Approximations between fuzzy expert systems and neural networks. International Journal of Approximate Reasoning, 10, 1994, 63-73.

16. Bunke H. and Kandel A. Neuro-fuzzy pattern recognition. World Scientific Publishing Co., Singapore 2000.

17. Jelodar H. and Aramideh J.: Comparison AODV and DSDV routing protocols with using fuzzy logic in the MANET. Advances in Science and Technology Research Journal, 8(22), 2014, 19-25.

18. Goswami S. and Das C.B.: Performance analysis of AODV, DSR and DSDV in MANET using NS-2. Global Journal of Computer Science and Information Technology, 1(1), 2014, 75-80.

19. Goswami S., Das C.B., Joardar S.: Performance comparison of routing protocols of MANET using NS-2. Lap Lambert Academic Publishing.

20. Goswami S., Joardar S., Das C.B.: Reactive and proactive routing protocols performance metric comparison in mobile ad hoc networks using NS-2. International Journal of Advanced Research in Computer and Communication Engineering, 3(1), 2014, 4908-4914.

21. Goswami S., Das C.B., Joardar S. Comparative performance analysis of DSDV and AODV routing protocols in MANET using NS-2. Networking and Communication Engineering. 5(12), 2013, 536-544. 